\title{
Nano- and microfluidic channels as electrokinetic sensors and energy harvesting devices
}

\author{
Pál Árki, Christine Hecker, Frederic Güth, Yvonne Joseph ${ }^{1}$ \\ ${ }^{1}$ Institute of Electronic and Sensor Materials, Technische Universität Bergakademie Freiberg, \\ Gustav-Zeuner-Str. 3. D-09599, Freiberg, Germany \\ Pal.Arki@esm.tu-freiberg.de
}

\begin{abstract}
Electrokinetic phenomena in charged micro- and nanochannels known as streaming potential and streaming current arise when pressure-driven fluid flow transport by advection induces both a potential and a current at the channel ends. This effect can be used as a sensor/transducer principle and also to generate electricity for sensor power supply and therefore represents a mechanism for converting mechanical work into electrical power. Electrokinetic sensors and energy harvester are simple to build, and can be easily integrated into nano- and microfluidic lab-on-chip systems.
\end{abstract}

Key words: nano- and microfluidic channels, electrokinetic, streaming potential, energy harvesting

\section{Introduction}

Micro- and especially nanofluidics is a young discipline that deals with fluid flow in narrow channels (Fig. 1.). In the last decades, synthetic nano/microcapillary structures constitute a new generation of devices for example in micro- and nano-enginieered lab on a chip and fluidic MEMS devices [1].

Controlled and precise fabrication of nano/microchannels, modification and functionalization of nano/microchannel surfaces and the use of external gate voltage control can lead to many new and unique applications of nano/microchannels such as sensors, nanofluidic transistors and diodes, ion pumping and energy harvesting devices [2,3,4]. Energy harvesters are neccesery for developing low power battery free sensors, and it is an interesting topic also for mobile and healthcare applications.

In the recent trend of miniaturization of flow devices, the surface-to-volume ratio will increase. In nano- and microfluids Interesting phenomena appears at the solid/liquid interfaces, different to the ones in macrofluidics. The surface charges of the nano/microfluidic channels (membranes, technical nano- and microchannels) result in a number of electric double-layer-related phenomena that occur at the solid-liquid interface.

In nano/microfluidic channels electrokinetic phenomena, such as electroosmosis and streaming potential, arise based on the charges of the inner capillary surface.
In electroosmosis, the driving force is an external electrical force which leads to a mechanical motion (actuator principle), and in streaming current or streaming potential (generator or sensor) mode the driving force is an applied mechanical force (hydrodynamic pressure) which produces an electric current or electric potential (transducer or generator principle).

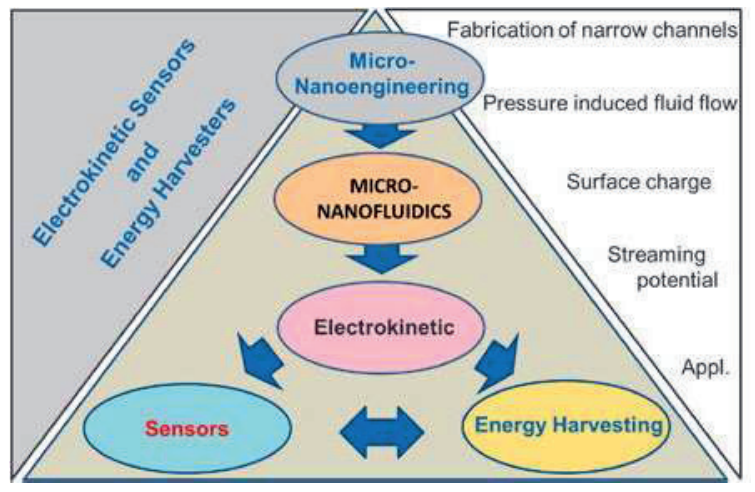

Fig. 1. Classification and contex of relevant disciplines and scientific and application fields Electrokinetic Sensors and Energy Harvesters

E.g. by using an electroosmosis pump a fluid in a lab-on-chip channel can be moved. Electrokinetic sensors are based on the reverse effect, the streaming potential (Fig. 2.).

Streaming potential electrokinetic sensors are similar to sensors based on the piezoelectric effect. Both sensors are active sensors; both generate electric signals without the need of any additional energy source (Fig. 3.). 
In the streaming potential process, the salt solution is forced by an external pressure to flow through a porous plug material, a single capillary respectively a capillary bundle or across a channel formed between two plates (Fig. 2).

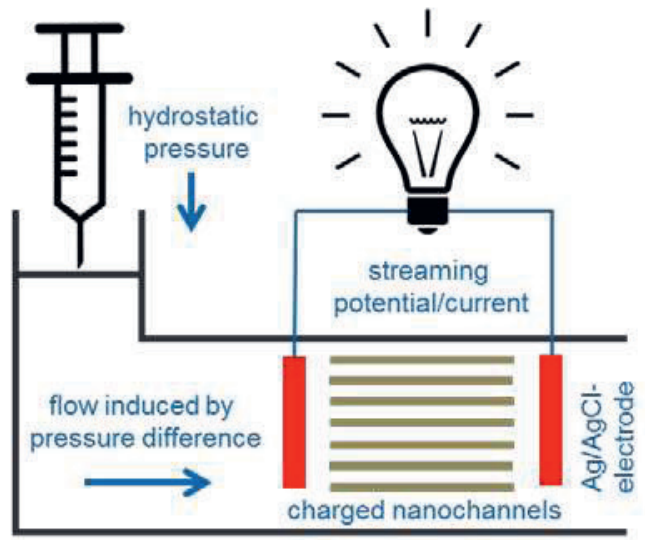

Fig. 2. Origin of fluid flow induced electrokinetic streaming potential -electrokinetic transducer/ power generator.

The streaming potential/current transducer or energy conversation effect is, - similar to piezoelectric effect (Fig. 3.), easy to generate with a pressure induced electrolyte flow. To measure, are only two silver/silver chloride electrodes and a high impedance volt/ampere meter need.

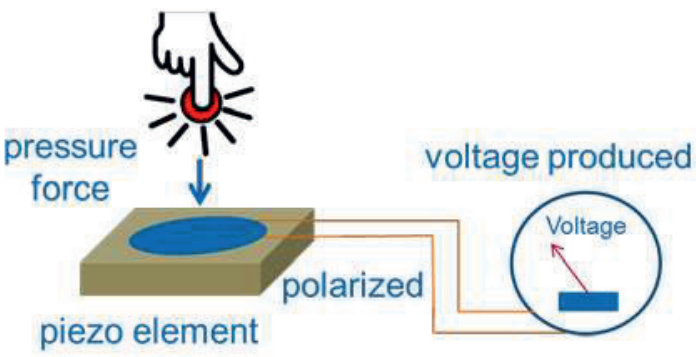

Fig. 3. Active piezoelectric sensors/harvester generate electric signals/power in response to an external stimulus (compare electrokinetic sensor/harvester in Fig.2).
Electrokinetic sensors based on streaming potential/current can detect the adsorption of chemicals, drugs or biological markers as well as pressure/flow or $\mathrm{pH}$ value. Electrokinetic sensors are active self-powered sensors, simple to build, and can be easily integrated into nano- and microfluidic lab-on-chip systems.

\section{Surface charge at the solid/liqid interface}

The basis of the electrical double layer related phenomena are that the walls of the fluid filled capillaries are charged. Surface charge is caused by charged function groups. These groups can be protoned and deprotoned depending on $\mathrm{pH}$ value and the sign of surface potential can change. Most metal oxid (ceramic) surfaces E.g. are amphoteric and have an isoelectric point (Fig. 4.). The surface chemistry can be modelled by the site binding model.

The surface charge will be compensated with co-ions and a so called electrical double layer will be built (Fig. 4. left). In nanochannels the Debay-length (the thickness of double layer) is the same dimension as the pore or channel radius. An overlapping of double layer and Donnan co-ion exclusion are caused (Fig. 4. center). This effect increases the sensitivity of the sensor and the efficiency of energy conversion. By the streaming potential effect forcing a liquid through a capillary or porous plug induces a difference of electric potentials because the ions of the diffuse part of the electrochemical double layer shift.

Zeta Potential is the potential at the hydrodynamic shear plate between solid and liquid surfaces. The zeta potential, which can be calculated from the measured streaming potential, represents the surface charge which occurs in the presence of an aqueous solution when functional groups dissociate on a surface or ions adsorb onto surfaces from the solution (Fig.4 right). In this way (change the sign and value of the zeta potential), electrokinetic or streaming potential sensors can detect the adsorption of chemicals or biological markers.

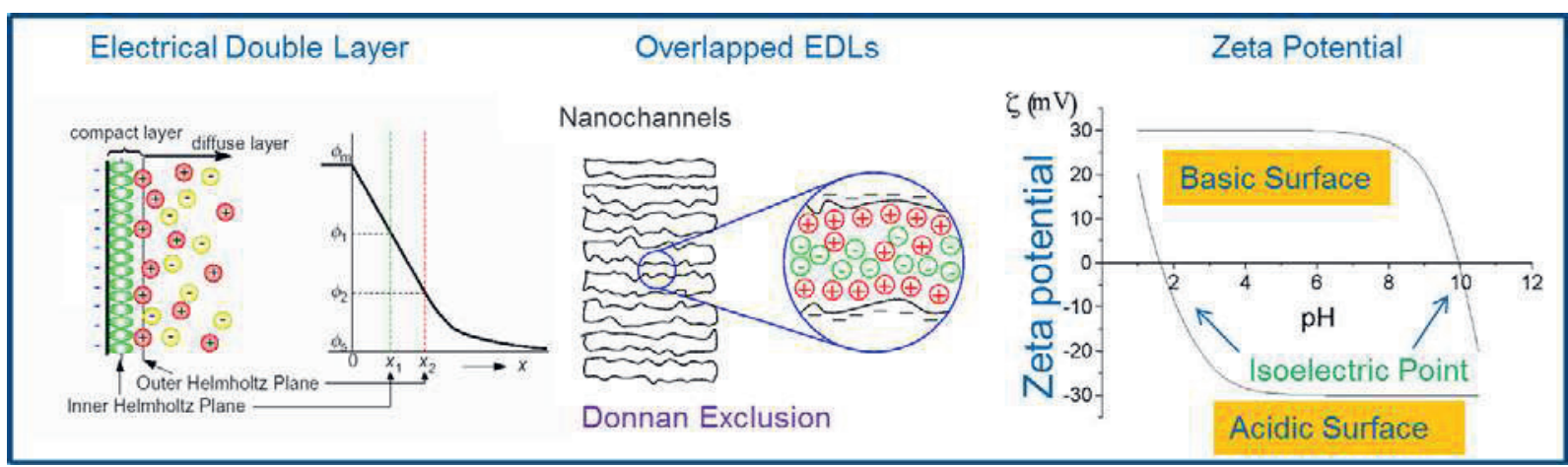

Fig. 4. Electrical Double Layer, Overlapping of EDLs, Zeta potential as a function of the $\mathrm{pH}$ value of the electrolyte respectively adsorption/desorption state of functional groups of the solid surfaces 


\section{Aims}

The streaming potential is a universal transducer principle, that we can use as a physical pressure sensor when alone, or can be combined with chemically specific layers (chemical interaction with channel walls) or receptors and become chemical or biological sensors. The dependencies of the streaming potential are expressed in the HelmholtzSmoluchowski equation (Eq.1). Hence, it is possible to develop physical sensors for pressure or flow measurements. Electrokinetic sensors are active sensors and generate electric signals in response to an external stimulus without the need of an additional energy source. The effect can also be understood as an energy conversion, and was investigated in energy harvesting applications as an electrokinetic power generator or battery.

The efficiency of electrokinetic energy conversion is very low (Tab. 1.) and should be increased by optimizing the complex effects at the solid-liquid interfaces in micro- and nanofluidic channels. The harvested electrokinetic energy could then provide power to battery-less wireless electrokinetic sensors e.g. in industrial pipeline systems

\section{Materials and measurement setup}

The sensor properties were examined in a selfdeveloped streaming potential measuring setup (Fig. 5.) A single micro- and nanocapillary or capillary system as the sensor element is housed in a measuring cell with ports for the required fluidic and electrical connections. The $\mathrm{Ag} / \mathrm{AgCl}$ electrodes were put in the vicinity of the end faces of the capillary sensor element. Fluid handling, including rinsing and measuring steps, was performed by the high pressure syringe pump. The pressure differences across the sample were created by pumping the salt solution through a capillary and were measured between two pressure transducers placed at the inlet and the outlet of the fluid flow. Both electrodes are connected to a high impedance voltmeter which records the current-less electrical potential (streaming potential). The entire setup is fully automated and controlled by a computer running a Visual Basic program.

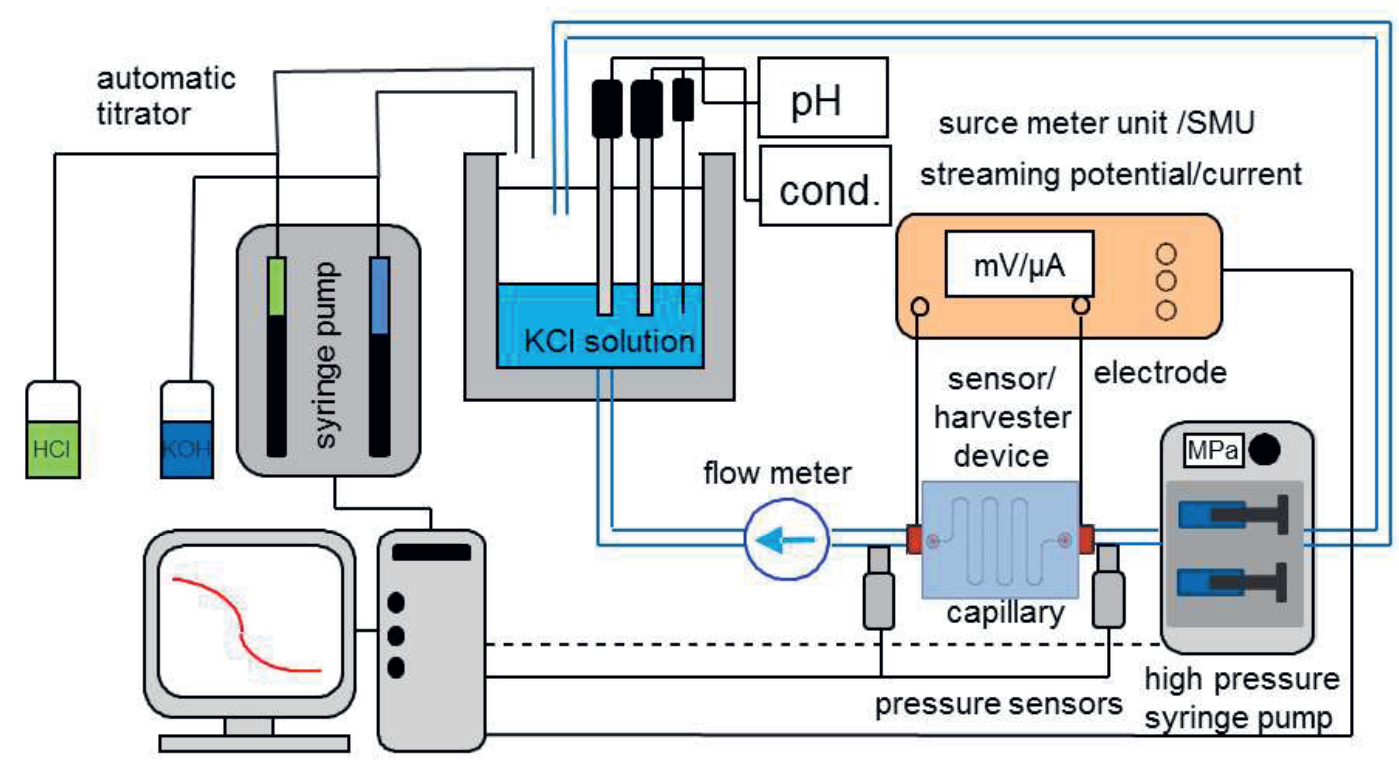

Fig.5. Schematic of the measurement setup for testing of electrokinetic sensors properties and energy harvesting efficiency, composed of an automatic $\mathrm{pH}$ titrator, external $\mathrm{pH}$ and conductivity electrodes with a temperature sensor, pressure sensors, a flowmeter, a measuring cell with sensor element/energy harvester and $\mathrm{Ag} / \mathrm{AgCl}$ electrodes on each end of the cell, a high impedance volt/amperemeter, two syringe pumps and a Visual Basic control and evaluation software.

Tab. 1: Some examples of efficiencies of electrokinetik energy harvesting channels and capillaries *Entries in italics are calculated from theoretical models without experimental verification

\begin{tabular}{|l|l|l|l|}
\hline Channel structure & Channel dimension & Max. power & Max. efficiency \\
\hline Porous alumina & $200 \mathrm{~nm}$ & $\sim 24 \mu \mathrm{W} / \mathrm{cm}^{3}$ & $0.77 \%[5]$ \\
\hline Single silica channel & $75 \mathrm{~nm}$ & $\sim 60 \mu \mathrm{W} / \mathrm{cm}^{3}$ & $3.2 \%[6]$ \\
\hline${ }^{*}$ Glass filter & $500 \mathrm{~nm}$ & - & $12 \%$ calculated [7] \\
\hline
\end{tabular}



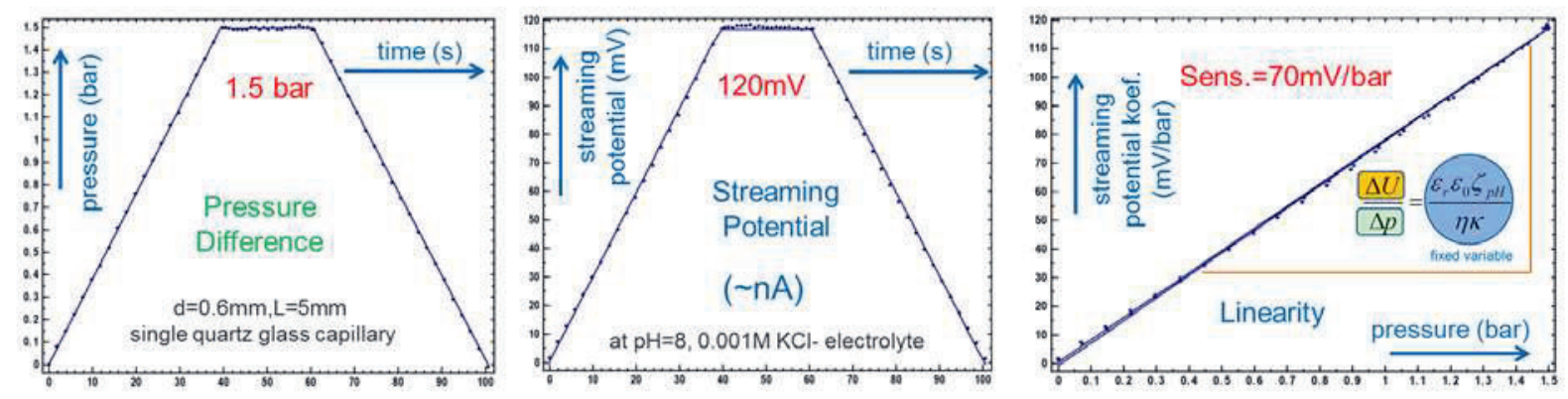

Fig. 6. Sensor signal/ generated voltage of elektrokinetic pressure sensors/harvester dependend on pressure difference between the ends of the quartz glass capillary (high sensitivity, good linearity, small offset, acceptable energy conversation-efficiency and power density)

\section{Measurement routines}

The pump transported a $\mathrm{KCl}$ electrolyte solution $(0.001 \mathrm{M})$ from a beaker kept at $25^{\circ} \mathrm{C}$ through the sensor testing cell, while measuring the conductivity, the temperature and the $\mathrm{pH}$. The applied pressure was increased during the measurement from 0 bar to a maximum differential pressure of 1.5 bar. The maximum attainable pressure difference is 6 bar. After pressure increase (upward measurement) and holding the pressure (plateau measurement), the pressure was reduced again (downward measurement) (Fig. 6. left) while measuring the streaming potentials (Fig. 6. centre). The streaming potential coefficient $(\Delta U / \Delta p)$ was calculated by the slopes of the linear pressurestreaming potential curve (upward and downward) at a given $\mathrm{pH}$ value (Fig. 6. right). For the analysis of the surface charge properties streaming potential measurements are performed, starting at $\mathrm{pH} 9$ followed by an acidification to $\mathrm{pH} 3$ and the corresponding zeta-potential values are obtained. All generated data is transmitted to the process control system. The zeta potential ( $\zeta$-potential) of the capillary surface can be calculated from the streaming potential $(\Delta U)$ using the Helmholtz-Smoluchowski equation eg.(1)

$$
\frac{\Delta U}{\Delta p}=\frac{\varepsilon_{r} \varepsilon_{0} \zeta_{p H}}{\eta \kappa}
$$

where $\Delta U / \Delta p$ (streaming potential coefficient) is the slope of the streaming potential versus hydraulic pressure curve, $\varepsilon_{0}$ is the permittivity of vacuum, $\varepsilon_{r}$ is the relative permittivity of the solvent, $\eta$ is the dynamic viscosity of the solution and $\mathrm{k}$ is the conductivity of the solution.

\section{Results and discussion}

The fluid flow induced electrokinetic effect (streaming potential) depends on its driving force (pressure difference) and the nature of solid (functional groups) and liquid phases $(\mathrm{pH}$ value, concentration of adsorbents and ions).
The streaming potential increases linearly with the fluid pressure difference that drives the fluid flow (Fig. 6). In this way, active pressure/flow sensors can easily be developed. The sensor element is in the simplest case a capillary or even the micro- nanofluidic cannel in a lab on chip system. The linearity of the sensor is given in the investigated pressure range ( $0-6$ bar).

Elektrokinetic pressure sensors have high sensitivity, good linearity, no or small offset. Offset appears only in nanochannels if ions are excluded by size and charge and a concentration difference between both ends of the channel takes place. Drift and Hysterises can be eliminated if we encapsulate it by a membrane and keep the surface charge constant.

Offset errors are negligible, and can be attributed to polarization phenomena and asymmetry potential (max. $5 \mathrm{mV}$ ) of the electrodes. The sensor response to the pressure/flow change is fast.

According to Helmholtz-Smoluchowski equation (Eq. 1) the sensitivity and harvested voltage/current is inversely proportional to the fluid conductivity and proportional to the surface charge (zeta potential at the shear plane of electrical double layer). A high sensitivity and energy conversation rate can be achieved in a dilute electrolyte solution with a capillary formed from a material with a high surface charge density.

Another possibility to increase the surface charge density and thereby the zeta potential and sensitivity/harvested voltage is the addition of specific adsorbent such as charged molecules (surfactants) or ions as well as adjusting the $\mathrm{pH}$ value to maximize the streaming potential coefficient. E.g. the specific adsorption of calcium and sulphate ions change the isoelectric point and increases the magnitude and change the sign of streaming potential signals in dependency of $\mathrm{pH}$-value. 
Tab.2: Some examples of existing energy harvesting approaches

\begin{tabular}{|c|c|c|}
\hline technology & performance & note/examples \\
\hline photovoltaic & $\begin{array}{c}100 \mu \mathrm{W} / \mathrm{cm}^{2} \\
\text { (illuminated } \\
\text { office) }\end{array}$ & $\begin{array}{c}40.7 \% \\
\text { efficient solar } \\
\text { cell }\end{array}$ \\
\hline piezoelectric & $\begin{array}{c}4 \mu \mathrm{W} / \mathrm{cm}^{3} \\
\text { (human } \\
\text { sources) }\end{array}$ & $\begin{array}{c}\text { Shoe- } \\
\text { mounted } \\
\text { harvesting, } \\
8.4 \mathrm{~mW}\end{array}$ \\
\hline thermoelectric & $\begin{array}{c}31 \mu \mathrm{W} / \mathrm{cm}^{2} \\
\text { at } \Delta \mathrm{T}=20 \mathrm{~K} \\
1 \mu \mathrm{W} / \mathrm{cm}^{2} \text { at } \\
\Delta \mathrm{T}=5 \mathrm{~K}\end{array}$ & $\begin{array}{c}\text { efficieny is } \\
\text { limited at } \\
\text { small } \\
\text { temperature } \\
\text { difference }\end{array}$ \\
\hline $\begin{array}{c}\text { electromagnetic } \\
\text { (amient RF) }\end{array}$ & $<1 \mu \mathrm{W} / \mathrm{cm}^{2}$ & $\begin{array}{c}\text { close } \\
\text { proximity to an } \\
\text { RF transmitter }\end{array}$ \\
\hline kinetic (airflow) & $2 \mathrm{~mW} / \mathrm{cm}^{3}$ & $\begin{array}{c}\text { Microturbine } \\
\text { electrokinetic } \\
\text { (liquid flow) }\end{array}$ \\
\hline
\end{tabular}

\section{Summary and conclusions}

The streaming potential can use as a characterization method of surface charges (zeta potential, isoelectric point) as well as a transducer (pressure sensor, affinity sensor) or energy harvesiter. The results of sensor characteristics tests show good linearity and high sensitivity for these sensors.

The electrokinetic energy conversation efficiency, power density and maximal harvestable voltage/current value on selected and suitable capillary systems were examined.

In our laboratory we tried to create enough power to function a calculator LCD display. We used two ceramic rods in which we pushed diluted electrolyte fluid at 6 bar pressure. The rods were connected hydrodynamically parallel and electrically serial so as to increase energy density and power. The efficiency was low, so for a real application, we need to research on increasing efficiency to be able to use it and make it economical.

The challenge for practical battery-less applications is the improvement of the electrokinetic energy conversion efficiency, which is currently low but still almost comparable with existing energy harvesting approaches (Tab.2.) like thermo- or piezoelectric harvesters.

\section{Outlook}

By external modulation of the surface charge by applying of an electric field, the charge-based surface effects in a nanofluidic channel are specifically reinforced. Sensitivity of sensors and efficiency of the energy harvesting applications can be controlled and improved. In the future, one can imagine that electrokinetic harvesters provide sensors with enough energy to send sensor results to a cloud via wireless technologies. Such sensors could be used in the fourth industrial revolution.

\section{References}

[1] S. Pennathur, J. Eijkel, and A. van den Berg, Energy conversion in microsystems: is there a role for micro/nanofluidics? Lab Chip 7, 1234-37 (2007); doi: 10.1039/B712893M

[2] T. Tsukahara , K. Mawatari and T. Kitamori: Integrated extended-nano chemical systems on a chip; Chem. Soc. Rev., 2010,39, 1000-13; doi: 10.1039/b822557p

[3] D. Kim, Pressure Sensor Using Electrokinetic Energy Conversion Phenomena, Sensors, 2007 IEEE

[4] Chih-Chang Chang and Ruey-Jen Yang: Electrokinetic energy conversion efficiency in ionselective nanopores Appl. Phys. Lett. 99, 083102; doi 10.1063/1.3625921

[5] Lu, M.C., Satyanarayana, S., Karnik, R., Majumdar, S., Wang, C.: „A mechanicalelectrokinetic battery using a nano-porous membrane", J. Micromech. Microeng. 16, 667675, (2006), doi: 10.1088/0960-1317/16/4/001

[6] van der Heyden, F. H. J., Stein, D., Dekker, C.: Streaming Currents in a single nanofluidic Channel", Phys. Rev. Lett. 95, 116104, (2005).doi:https://doi.org/10.1103/PhysRevLett.9 5.116104

[7] van der Heyden, F. H. J., Bonthuis, D. J., Stein, D., Meyer, C., Dekker, C.: „Elektrokinetic Energy Conversion Efficiency in Nanofluidic Channels", Nano Lett. 6 (10), 2232-2237,(2006).doi: $10.1021 / \mathrm{nl} 061524 \mid$ 